\title{
Antibodies to Shiga toxin in the serum of children with Shigella-associated haemolytic uraemic syndrome
}

\author{
T. AZIM, A. RASHID*, F. QADRI, M. S. SARKER, J. HAMADANI, M. A. SALAM, M. A. WAHED \\ and M. J. ALBERT
}

International Centre for Diarrhoeal Disease Research, Bangladesh (ICDDR,B) GPO Box 128, Dhaka 1000 and * Department of Microbiology, Institute of Postgraduate Medicine and Research, University of Dhaka, Dhaka, Bangladesh

\begin{abstract}
Antibodies to Shiga toxin (Stx) were measured in the sera of 49 children with Shigella dysenteriae serotype 1 infection, of whom 17 had haemolytic uraemic syndrome (HUS) and 32 had no complications (uncomplicated shigellosis, UCS). Children with HUS had lower levels of total IgG and IgM and lower IgM titres to Stx than those with UCS. The number of children with neutralising antibodies was similar in the two groups. Of the children with HUS, 11 had HUS on enrolment and six developed HUS subsequent to enrolment. Antibody titres in children who subsequently developed HUS were compared with those in children with UCS to assess whether differences in antibody titres occurred before the development of HUS. IgA titres to Stx were found to be higher in children who subsequently developed HUS than in those with UCS. However, logistic regression analysis revealed that titres of Stx antibodies in the serum were not significant risk factors for the development of HUS. Thus, although the levels of Stx antibodies were different in children with HUS, and higher IgA titres to Stx were identifiable in children who subsequently developed HUS compared with those with UCS, the relevance of these findings in the development of HUS remains to be elucidated.
\end{abstract}

\section{Introduction}

Haemolytic uraemic syndrome (HUS) is a triad of haemolytic anaemia, thrombocytopaenia and acute renal failure that often complicates dysentery following infection with Shigella dysenteriae serotype 1 [1] or Shiga toxin-producing Escherichia coli (STEC) [2]. The mechanism(s) whereby these bacteria precipitate HUS is not understood, but Shiga toxin (Stx) produced by $S$. dysenteriae 1, and Stx 1 and Stx 2 (also known as SLT-I and SLT-II, respectively) produced by STEC appear to play a pivotal role. Stx and Stx1 are closely related and the base sequence of their genes differs by only three nucleotides [3]. It is not clear how these toxins induce HUS, but Stx and Stx2 can damage vascular endothelial cells in vitro $[4,5]$ either alone or in combination with cytokines and the bacterial lipopolysaccharide (LPS). More recently it has been

Received 2 March 1998; accepted 9 April 1998. Corresponding author: Dr T. Azim. shown that injection of Stx2 in mice mimics the glomerular changes observed in HUS [6]. The importance of these toxins in the development of HUS has led to the hypothesis that antibodies to these toxins could prevent HUS. Serum antibodies to Stxl and Stx2, neutralising as well as total immunoglobulin (Ig) isotypes, have been described in patients with STEC-associated HUS [7-10], but the titres were not as high or as consistent as those to the LPS [11]. Commercially available human intravenous immunoglobulin (IVIG) preparations contain neutralising antibodies to Stx and Stx1, but not Stx2 [12], and the use of IVIG in a trial of a limited number of children with HUS showed a beneficial effect [13]. Furthermore, human IVIG given to rabbits either before or within $1 \mathrm{~h}$ of Stx1 or Stx2 injection protected rabbits from developing diarrhoea due to Stx1 but not Stx2 [14]. Therefore, immunisation with toxoid for prevention of HUS in children infected with $S$. dysenteriae 1 or STEC [15] appears to be promising. However, the role of antibodies to Stx in protection against shigellosis and the development of HUS is unclear. Bangladeshi 
patients with uncomplicated $S$. dysenteriae 1 infection elicit a strong antibody response to Stx with both IgA1 and IgG1 levels peaking at 9-11 days after the onset of diarrhoea [16]. Furthermore, both IgA and IgG responses to Stx are greater in patients with more severe illness, suggesting that these antibodies reflect disease severity rather than protection [17]. The present study describes Stx antibody levels (IgA, IgG, IgM and neutralising) in the sera of children with $S$. dysenteriae 1 infection with and without HUS, and also compares antibody titres between children with uncomplicated shigellosis (UCS) and those who subsequently developed HUS.

\section{Materials and methods}

\section{Patient population}

Initially, 49 children between 12 and 60 months of age attending the Clinical Research and Service Centre of the International Centre for Diarrhoeal Disease Research, Bangladesh (ICDDR,B), Dhaka, with complaints of dysentery (visible blood in stool, with or without fever) were enrolled. Stool and blood samples ( $5 \mathrm{ml}$, maximum) were collected from these children on enrolment. Freshly collected stools were examined microscopically for the presence of white (WBC) and red blood cells (RBC) and were cultured for enteropathogens [18]. Only children with culture-confirmed $S$. dysenteriae 1 were finally included in this study.

Laboratory investigations on blood included determination of haematocrit, total and differential leucocyte counts, platelet counts and the proportion of fragmented erythrocytes. Serum electrolytes and creatinine concentrations were measured when required. All children were treated with amdinocillin pivoxil or ciprofloxacin, while some received additional antibiotics for concomitant infections such as respiratory tract infections, septicaemia or middle ear infections. HUS was defined as serum creatinine levels $\geqslant 180 / \mu \mathrm{mol}$ of blood, signs of haemolysis, estimated either by haematocrit values falling by $\geqslant 10 \%$ during hospitalisation or the presence of $\geqslant 0.5 \%$ fragmented erythrocytes on peripheral blood smears, and platelet counts of $<150000 / \mu$ l of blood. Of all the children enrolled, 17 had HUS and 32 had no complications including HUS, acute renal failure, leukaemoid reaction or toxic megacolon (UCS). Of the children with HUS, eight had leukaemoid reaction $(\geqslant 40000 \mathrm{WBC} / \mu \mathrm{l}$ of blood, increased percentage of neutrophils and presence of immature neutrophils). Of the 17 children with HUS, 11 had HUS at the time of enrolment while six developed HUS subsequent to enrolment. The nutritional status of all children was determined by weight-for-age as a percentage of the National Centre for Health Statistics (NCHS) median. The study was approved by the Ethical Review Committee of ICDDR,B and only those children whose guardians gave informed, written consent were enrolled.

\section{Collection and storage of serum and plasma samples}

Serum was obtained from blood collected in sterile tubes and stored at $-20^{\circ} \mathrm{C}$ until used. Plasma was collected from fresh blood separated on Ficoll-Hypaque (Pharmacia, Uppsala, Sweden), in which plasma collected as a clear supernate above the band of mononuclear cells at the interface. It was filtered through a $0.22-\mu \mathrm{m}$ pore size filter (Sartorius, Goettingen, Germany) and stored at $-20^{\circ} \mathrm{C}$ until tested.

\section{Determination of total immunoglobulins}

Total IgA, IgG and IgM levels in plasma were measured by turbidimetry in a discrete analyser (COBAS-BIO; Roche) with monospecific antisera (Dakopatts, Glostrup, Denmark). Results were expressed as $\mathrm{mg} / \mathrm{ml}$ of plasma.

\section{Measurement of antibodies to Stx 1 by enzyme- linked immunosorbent assay}

Specific antibody titres to Stx1 were measured by an enzyme-linked immunosorbent assay (ELISA) as described previously [16]. Microtitration plates (Maxisorp, Nunc, Roskilde, Denmark) were coated with $100 \mu \mathrm{l} /$ well of purified Stx1 (a gift from Dr D. W. Acheson and Professor G. T. Keusch, New England Medical Center, Boston, MA, USA) [19] at $1.5 \mu \mathrm{g} / \mathrm{ml}$ in phosphatebuffered saline (PBS)-Tween 20 and incubated overnight at $4^{\circ} \mathrm{C}$. After blocking with PBS-Tween containing bovine serum albumin (BSA) $0.1 \%$, serum samples, serially diluted in PBS-Tween containing BSA $0.1 \%$, were added in duplicate to wells and incubated at $37^{\circ} \mathrm{C}$ for $2 \mathrm{~h}$. After washing, horseradish peroxidase conjugated to rabbit anti-human IgA, IgM or IgG (Jackson ImmunoResearch Laboratories, West Grove, PA, USA) diluted 1 in 1000 in PBS-Tween containing BSA 0.1\%, was added and plates were incubated at $37^{\circ} \mathrm{C}$ for $1 \mathrm{~h}$. The substrate, $o$-phenylenediamine dihydrochloride (OPD, Sigma) at $1 \mathrm{mg} / \mathrm{ml}$ in $0.1 \mathrm{M}$ citrate buffer $(\mathrm{pH}$ 4.5)-hydrogen peroxide (Sigma) 30\%, was added, and the reaction was stopped with $1 \mathrm{M} \mathrm{H}_{2} \mathrm{SO}_{4}$ after colour development. The OD was measured at $492 \mathrm{~nm}$ in a spectrophotometer (Titertek Multiskan Plus). Results were expressed as end-point titres which were determined as the interpolated dilution of the sample giving an $\mathrm{OD}_{492}$ of 0.2 above background. Background wells included those without samples. Calculations were done with a computer-based program (Multi; DataTree, Watthams, MA, USA).

\section{Neutralisation assay}

Antibody neutralisation titres were determined by the HeLa cell cytotoxicity assay [20]. Periplasmic extract (PE) was prepared from $S$. dysenteriae 1 strain 24583 (obtained from an adult patient with UCS) with polymyxin B [21] and used as a source of Shiga toxin. Briefly, HeLa cells at $2 \times 10^{4}$ cells $/ \mathrm{ml}$ of minimal 
essential medium (MEM) containing fetal bovine serum (FBS; Gibco, Grand Island, NY, USA) 10\% were grown as monolayers in flat-bottomed 96-well microtitration plates (Nunc) for $18-20 \mathrm{~h}$ at $37^{\circ} \mathrm{C}$ in a $\mathrm{CO}_{2} 5 \%$ incubator. Serial two-fold dilutions of serum samples in MEM containing FBS $10 \%$, from 1 in 4 to 1 in 64 , were pre-incubated for $3 \mathrm{~h}$ with medium alone or PE diluted 1 in 8000 in MEM containing FBS $10 \%$ (which resulted in $\sim 60 \%$ cytotoxicity of HeLa cells). A negative control of $\mathrm{PE}$ with medium and a positive control of PE with a neutralising anti-Stx monoclonal antibody (ICT7) [20] were always included in the assays. These were added in duplicate to HeLa cells and incubated for $18-20 \mathrm{~h}$. Detached cells were removed and the remaining cells were fixed with formalin 5\% in PBS, stained with crystal violet $0.13 \%$ in PBS containing ethanol $50 \%$ and formalin $2 \%$, washed and dissolved with absolute ethanol. Optical densities were measured at $595 \mathrm{~nm}$ with a spectrophotometer (Titertek Multiskan Plus). Neutralisation of cytotoxicity by $\geqslant 20 \%$ was considered as a positive response as described previously [12].

\section{Statistical analyses}

The significances of differences between two groups of children were ascertained by the $t$ test (for continuous, parametric data) and the Mann-Whitney $U$ test (for continuous, non-parametric data). The $\chi^{2}$ test was used to compare proportions. Multiple regression analysis was used to assess the effect of nutritional status, duration of diarrhoea on enrolment, stool frequency in $24 \mathrm{~h}$, sex and concomitant infections on Stx antibody titres. Logistic regression was used to determine whether serum Stx antibodies were risk factors for the development of HUS. Differences were considered to be significant when $\mathrm{p} \leqslant 0.05$. Data were analysed with the Statistical Package for Social Sciences (version 6.0 for Windows, SPSS, Chicago, IL, USA).

\section{Results}

\section{Patient population}

The clinical characteristics of the children studied are shown in Table 1. Children in the two groups were comparable for age, sex, nutritional status, duration of diarrhoea on enrolment and the state of hydration (data not shown). Routine laboratory investigations (Table 1) showed higher numbers of $\mathrm{WBC} / \mu \mathrm{l}$ of blood $(p<0.001)$ and higher percentages of polymorphonuclear cells $(p=0.001)$ and myelocytes $(p=0.001)$ in children with HUS when compared with those with UCS. These findings are not surprising, as 8 of 17 also had leukaemoid reaction, and are similar to previous reports where leukaemoid reaction accompanied most cases of HUS [1]. Compared with children with UCS, those with HUS had fewer platelets in blood $(p=0.001)$ and a greater percentage of fragmented erythrocytes $(p<0.001)$; these findings are consistent with the definition of HUS.

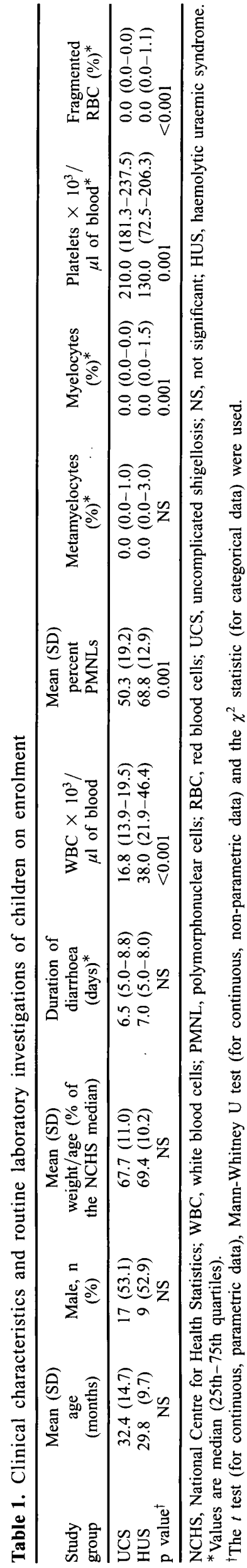


Total immunoglobulins, titres of antibodies to Stx and neutralisation titres

Comparison of total IgA, IgG and IgM levels in plasma of children with HUS with those with UCS, showed that IgG and IgM levels were lower in children with HUS $(\mathrm{p}<0.001$ and $\mathrm{p}=0.036$, respectively, Table 2) while total IgA levels were similar. Serum IgM titres to Stx were lower in children with HUS $(p=0.032)$ than in those with UCS, while Stx IgG and IgA titres were similar in the two groups of children (Table 2). Because it is known that various clinical parameters may influence the immune response, a multiple regression analysis was performed to assess the effect of nutritional status, stool frequency in the $24 \mathrm{~h}$ before admission, duration of diarrhoea before enrolment, sex and concomitant infections on Stx antibody titres. None of these parameters had any effect on Stx antibody titres.

The numbers of children with serum neutralising antibodies to Stx were similar in the two groups of children (Table 2).

Comparison of children with UCS and those who subsequently developed HUS

Of the children with HUS, 11 had HUS on enrolment and six developed HUS after enrolment (median 3.5 days, range 1-6 days). Children who subsequently developed HUS and those with UCS were compared to assess whether changes in antibody levels were identifiable before the onset of HUS (Table 3). The two groups of children were similar in terms of sex, nutritional status (data not shown) and duration of diarrhoea before enrolment. Children who subsequently developed HUS had higher total WBC counts in blood $(p=0.003)$, and a higher percentage of polymorphonuclear cells $(p=0.037)$ and RBC fragments $(p=$ 0.021 ) in blood than children with UCS (Table 3). Although these high levels were higher than normal, none of the children who subsequently developed HUS had a leukaemoid reaction at the time of enrolment. Of the total Ig levels, IgG was lower in children who subsequently developed HUS (median $4.2 \mathrm{mg} / \mathrm{ml}$, 25th-75th quartiles 3.8-7.8) compared with those with UCS (median $8.0 \mathrm{mg} / \mathrm{ml}$, 25th-75th quartiles $6.4-11.7 ; \mathrm{p}=0.009$ ). Comparison of $\mathrm{Stx}$ antibody titres showed higher $\operatorname{IgA}$ titres in children who subsequently developed HUS ( $p=0.045)$, while there were no differences in Stx IgG and IgM titres (Table 3). Logistic regression to assess whether Stx antibody titres were risk factors for the development of HUS showed no significance.

\section{Discussion}

The role of Shigella-specific antibodies in the development of HUS is unclear. Higher antibody titres to homologous LPS have been described in the plasma of 
children with complicated shigellosis (including HUS) than in children with uncomplicated shigellosis (UCS) [22]. Antibodies to Stx in Shigella-associated HUS have not been described. In adults with UCS, Stx antibody levels have been shown to correlate with disease severity [17]. Whether these findings suggest that more Stx is produced in children who are more severely ill with $S$. dysenteriae 1 infection is not known. In this study, Stx antibody titres were determined in the serum of children with Shigellaassociated HUS and compared with those in children with UCS. Also, the Stx antibody titres were compared in children with UCS and those who subsequently developed HUS to assess whether changes in Stx antibody titres were associated with the development of HUS.

Studies on adults with UCS have shown that total IgG levels are lower in sera but higher in the stools of patients with shigellosis compared with healthy controls, suggesting that low levels in serum are secondary to increased loss in the stool [16]. Although IgA was also lost in the stool, in the same patients, serum IgA levels were not lowered [16]. This is possibly because locally, in the rectal mucosa, there is increased production of IgA [23]. In the present study, children with HUS had similar plasma levels of IgA, but lower levels of $\operatorname{IgG}$ and $\operatorname{IgM}$ than children with UCS. The lower IgG and IgM levels in plasma could be caused by increased loss in the stool, and the unaltered levels of plasma IgA may reflect increased production locally, as it has been shown that there are more IgA positive cells in the rectal mucosa in patients with greater inflammation [23]. Unfortunately, antibody levels in the stool were not measured in this study. Another possibility is direct inhibition of antibody secreting cells by Stx, as Stx has been shown to inhibit stem cells producing IgG- and IgAsecreting cells, but not IgM-secreting cells [24]. However, the finding of lowered IgG and IgM levels and similar IgA levels in children with HUS and UCS in the present study contradicts this suggestion. Also, differences in antibody levels in these two groups of children caused by the influence of Stx on production of antibodies suggest that the production of Stx is different in children with HUS and those with UCS. A preliminary and limited determination of Stx concentrations in stools from these children showed no differences between the two groups of children (unpublished observations).

Comparison of Stx antibody titres in the serum of children with UCS and HUS showed lower titres of Stx IgM in children with HUS, while the Stx IgG and IgA titres were similar in the two groups. Similar results have been reported from children with $E$. coli O157:H7-associated HUS [11]. The reason for this selective decrease in Stx IgM titres is not clear. One possibility is that children with HUS are experiencing a secondary infection. Moreover, it is not known 
whether IgM Stx antibodies have a role in protection against Shigella-associated HUS.

To assess whether antibodies in the serum are risk factors for the development of HUS, children with UCS were compared with those who developed HUS subsequent to enrolment. The latter had lower total IgG levels, which could be due to greater loss in the stool or to inhibition by Stx. Higher IgA Stx titres may be a reflection of a more severe illness in children with HUS compared with those with UCS, or secondary to stimulation by cytokines such as IL6, which is known to be elevated in the sera of children with HUS [25]. However, as logistic regression (carried out to assess whether Stx antibodies in the serum influenced the development of HUS), showed no significant association, the significance of different Stx antibody titres in the serum of children with HUS remains unclear.

In summary, differences in Stx antibodies occur in children with Shigella-associated HUS and some differences are observed before the development of HUS. However, the role of these antibodies in HUS remains to be elucidated.

This research was supported by the International Centre for Diarrhoeal Disease Research, Bangladesh, (ICDDR,B) and the US Agency for International Development grant DPE-5986-A-1009-00. ICDDR,B is supported by agencies and countries that share its concern for the health problems of developing countries. We thank Mr M. Haque for secretarial assistance and Drs A. Chowdhury and N. Khanum for help with patient enrolment.

\section{References}

1. Rahaman MM, Jamial Alam AKM, Islam MR, Greenough WB, Shiga bacillus dysentery associated with marked leukocytosis and erythrocyte fragmentation. Johns Hopkins Med J 1975; 136: $65-70$.

2. Karmali MA, Steele BT, Petric M, Lim C. Sporadic cases of haemolytic-uraemic syndrome associated with faecal cytotoxin and cytoxin-producing Escherichia coli in stools. Lancet 1983; 1: $619-620$.

3. Acheson DWK, Levine MM, Kaper JB, Keusch GT. Protective immunity to Shiga-like toxin I following oral immunization with Shiga-like toxin 1 B subunit-producing Vibrio cholerae CVD 103-HgR. Infect Immun 1996; 64: 355-357.

4. Louise CB, Obrig TG. Shiga toxin-associated hemolytic uremic syndrome: combined cytotoxic effects of Shiga toxin and lipopolysaccharide (endotoxin) on human vascular endothelial cells in vitro. Infect Immun 1992; 60: 1536-1543.

5. Obrig TG, Del Vecchio PJ, Brown JE et al. Direct cytotoxic action of Shiga toxin on human vascular endothelial cells. Infect Immun 1988; 56: 2373-2378.

6. Pulimood A, Koshi R, Date A, Mathan M. A murine model for haemolytic uremic syndrome, abstr. V105/V. In: VTEC '97 3rd International Symposium and Workshop on Shiga Toxin (Verocytotoxin)-Producing Escherichia coli Infections, 1997: Baltimore, MD, USA, p. 86.

7. Caprioli A, Luzzi I, Rosmini F et al. Hemolytic-uremic syndrome and vero cytotoxin-producing Escherichia coli infection in Italy. $J$ Infect Dis 1992; 166: 154-158.

8. Karmali MA, Petric M, Winkler M et al. Enzyme-linked immunosorbent assay for detection of immunoglobulin G antibodies to Escherichia coli vero cytotoxin 1. J Clin Microbiol 1994; 32: 1457-1463.

9. Lopez EL, Diaz M, Grinstein S et al. Hemolytic uremic syndrome and diarrhea in Argentine children: the role of Shiga-like toxins. J Infect Dis 1989; 160: 469-475.

10. Yamada S, Kai A, Kudoh Y. Serodiagnosis by passive hemagglutination test and verotoxin enzyme-linked immunosorbent assay of toxin-producing Escherichia coli infections in patients with hemolytic-uremic syndrome. J Clin Microbiol 1994; 32: 955-959.

11. Greatorex JS, Thorne GM. Humoral immune responses to Shiga-like toxins and Escherichia coli 0157 lipopolysaccharide in hemolytic-uremic syndrome patients and healthy subjects. $J$ Clin Microbiol 1994; 32: 1172-1178.

12. Ashkenazi S, Cleary TG, Lopez E, Pickering LK. Anticytotoxin-neutralizing antibodies in immune globulin preparations: potential use in hemolytic-uremic syndrome. $J$ Pediatr 1988; 113: $1008-1014$.

13. Sheth KJ, Gill JC, Leichter HE. High-dose intravenous gamma globulin infusions in hemolytic-uremic syndrome: a preliminary report. Am JDis Child 1990; 144: 268-270.

14. Havens PL, Dunne WM, Burd EM. Effects of human intravenous globulin on diarrhoea caused by Shiga-like toxin I and Shiga-like toxin II in infant rabbits. Microbiol Immunol 1992; 36: 1077-1085.

15. Keusch GT, Acheson DWK, McIver J, Kaper JB. Active and passive immunoprophylaxis for thrombotic microangiopathy secondary to infection with Shiga toxin-producing Escherichia coli (Abstract) In: The thirty-second joint conference US-Japan co-operative Medical Science Program, Cholera and related diarrheal diseases panel, 1996, Nagasaki, Japan. p. 119.

16. Islam D, Wretlind B, Ryd M, Lindberg AA, Christensson B. Immunoglobulin subclass distribution and dynamics of Shigellaspecific antibody responses in serum and stool samples in shigellosis. Infect Immun 1995; 63: 2054-2061.

17. Islam D, Wretlind B, Hammarström L, Christensson B, Lindberg AA. Semiquantitative estimation of Shigella antigenspecific antibodies: correlation with disease severity during shigellosis. APMIS 1996; 104: 563-574.

18. World Health Organization. Manual for laboratory investigations of acute enteric infections. Geneva, World Health Organization. 1987: 9-20.

19. Donohue-Rolfe A, Acheson DWK, Kane AV, Keusch GT. Purification of Shiga toxin and Shiga-like toxins I and II by receptor analog affinity chromatography with immobilized Pl glycoprotein and production of cross-reactive monoclonal antibodies. Infect Immun 1989; 57: 3888-3893.

20. Qadri F, Mohi MG, Azim T, Faruque SM, Qadri SS, Albert MJ. Production, characterization and immunodiagnostic application of a monoclonal antibody to Shiga toxin. $J$ Diarrhoeal Dis Res 1996; 14: $95-100$

21. Downes FP, Green JH, Greene K, Strockbine N, Wells JG, Wachsmuth IK. Development and evaluation of enzyme-linked immunosorbent assays for detection of Shiga-like toxin I and Shiga-like toxin II. J Clin Microbiol 1989; 27: 1292-1297.

22. Azim T, Qadri F, Ahmed S et al. Lipopolysaccharide-specific antibodies in plasma and stools of children with Shigellaassociated leukemoid reaction and hemolytic-uremic syndrome. Clin Diagn Lab Immunol 1996; 3: 701-705.

23. Islam D, Veress B, Bardhan PK, Lindberg AA, Christensson B. Quantitative assessment of IgG and IgA subclass producing cells in rectal mucosa during shigellosis. J Clin Pathol 1997; 50: $513-520$

24. Cohen A, Madrid-Marina V, Estrov Z, Freedman MH, Lingwood CA, Dosch H-M. Expression of glycolipid receptors to Shiga-like toxin on human B lymphocytes: a mechanism for the failure of long-lived antibody response to dysenteric disease. Int Immunol 1990; 2: 1-8.

25. DeSilva DGH, Mendis LN, Sheron $\mathrm{N}$ et al. Concentrations of interleukin 6 and tumour necrosis factor in serum and stools of children with Shigella dysenteriae 1 infection. Gut 1993; 34: 194-198. 